\title{
Estrutura Fatorial da Escala de Respostas Comportamentais à Mudança Organizacional
}

\author{
Vanessa de Fátima Nery, Elaine Rabelo Neiva ${ }^{1}$, Kettyplyn Sanches Franco \\ Universidade de Brasília, Brasília-DF, Brasil
}

RESUMO

As respostas comportamentais são fundamentais para o sucesso das mudanças organizacionais. O presente estudo descreve a busca por evidências de validade do construto da Escala de Respostas Comportamentais à Mudança Organizacional, instrumento que integra duas dimensões comportamentais - Comportamento de Apoio e Resistência - na mesma escala. A escala também foi construída em duas versões: com o referente no nível individual e com o referente no nível coletivo. Para isso, foram realizadas análises fatoriais exploratórias $(N=239$ e $N=294)$ e análises fatoriais confirmatórias $(N=400$ e $N=424)$ das duas versões da escala e os resultados são comparados para discutir a questão do nível de análise. Os resultados corroboraram a pertinência de solução bifatorial, com 11 itens, apesar da correlação entre os fatores. A análise fatorial confirmatória ratificou os resultados encontrados na AFE. Quanto à precisão do instrumento, por meio dos coeficientes alfa de Cronbach, foram verificados bons índices de consistência interna.

Palavras-chave: mudança organizacional; análise fatorial; medida.

\section{ABSTRACT - Factor Structure of the Behavioral Responses to Organizational Change Scale}

Behavioral responses are critical to success in organizational change. The present study describes the search for construct validity evidence for the Behavioral Responses to Organizational Change Scale, an instrument that integrates two behavioral dimensions - supportive behavior and resistance - on the same scale. The scale was also constructed in two versions: one with the referent at the individual level and one at the collective level. To this end, exploratory factor analyses $(N=239$ and $N=294)$ and confirmatory factor analyzes $(N=400$ and $N=424)$ of the scale's two versions were performed and results are compared to discuss the level of analysis question. The results corroborated the pertinence of a two-factor solution, with 11 items, despite the correlation between the factors. Confirmatory factor analysis ratified the results found in EFA. As to the instrument's accuracy, good indices of internal consistency were verified through Cronbach's alpha coefficients,

Keywords: organizational change; factor analysis; measurement.

\section{RESUMEN - Estructura Factorial de la Escala de Respuestas Comportamentales al Cambio Organizacional}

Las respuestas comportamentales son fundamentales para el éxito de los cambios organizacionales. Este estudio describe la búsqueda de evidencias de validez del constructo de la Escala de Respuestas Comportamentales con relación al Cambio Organizacional, instrumento que integra dos dimensiones del comportamiento - comportamiento de apoyo y resistencia - en la misma escala. La escala también fue realizada en dos versiones: con referencia al nivel individual y con referencia al nivel colectivo. Fueron realizados análisis factorial exploratorio $(N=239$ y $N=294)$ y análisis factorial confirmatorio $(N=400$ y $N=424)$ de las dos versiones de la escala. Los resultados son comparados, para discutir el tema de nivel de análisis y los mismos corroboraron la pertinencia de solución bifactorial, con 11 ítems, a pesar de la correlación entre los factores. El análisis factorial confirmatorio ratificó los resultados encontrados en la AFE. En cuanto a la precisión del instrumento, por medio de los coeficientes alfa de Cronbach, se verificaron buenos índices de consistencia interna.

Palabras clave: cambio organizacional; análisis factorial; medida.

A mudança, definida como alterações na organização que possuem impactos nos membros ou nos resultados organizacionais, tornou-se processo contínuo, um aspecto inerente ao cotidiano organizacional (Neiva \& Paz, 2012). Dentre os critérios mais utilizados para avaliar as mudanças estão os dados objetivos, ou seja, valores numéricos relacionados à produtividade $\mathrm{e}$ ao resultado organizacional (Neiva \& Paz, 2012). As respostas comportamentais e afetivas, tais como resistência e comportamentos de apoio, também devem ser utilizadas como indicadores relevantes para aferir o sucesso das mudanças implantadas (Bordia, Restubog, Jimmieson, \& Irmer, 2011).

As respostas dos indivíduos à mudança têm atraído o interesse dos pesquisadores pelo seu impacto no sucesso do processo de mudança (Bordia et al., 2011; 
Oreg, Vakola, \& Armenakis, 2011; Vakola, Armenakis, \& Oreg, 2013; Vakola, 2016). Podendo envolver os aspectos cognitivo, afetivo e comportamental, as respostas dos indivíduos à mudança organizacional constituem reações emitidas pelos indivíduos durante os programas de mudança e apresentam valência positiva, negativa ou ambivalente (Piderit, 2000). Em geral a mudança organizacional pode eliciar diferentes emoções e reações que variam do otimismo ao medo, podendo incluir ansiedade, resistência, entusiasmo, incapacidade, motivação e pessimismo (Bordia et al., 2011; Bortolotti, 2010).

\section{Comportamentos de apoio à mudança organizacional}

Dentre os vários termos usados para se referir ao poio dos indivíduos ao processo de mudança, consta "comprometimento com a mudança" que é definido como "uma força (mind-set) que liga um indivíduo a um curso de ação considerado necessário para a implantação bem-sucedida de uma iniciativa de mudança" (Herscovitch \& Meyer, 2002, p. 475). Mais recentemente, muitos autores argumentam que enfatizar os comportamentos atuais, as contribuições ativas e as ações coletivas produzem maiores resultados de pesquisa que a ênfase em estados psicológicos, respostas passivas e individuais (Kim, Hornung, $\&$ Rousseau, 2011).

Comportamentos de apoio à mudança já foram considerados em estudos anteriores como a emissão de comportamentos desejados pelo processo de mudanças - de participação no processo - ou que facilitem, promovam as transformações (Kim et al., 2011). Nesse sentido, Kim, Hornung e Rousseau (2011) definem os comportamentos de apoio à mudança organizacional como as ações dos indivíduos que se engajam, comprometem-se no processo de mudanças e participam ativamente para aceitar, facilitar, contribuir e defender o processo de mudança planejada. A definição desses autores foi adotada neste estudo.

Essa definição contém três elementos que a diferenciam de outros construtos: (a) é centrada no comportamento real, ao invés de estados psicológicos relacionados às mudanças organizacionais, como, por exemplo, as atitudes ou intenções comportamentais; (b) enfatiza contribuições ativas para mudar, adaptando-se, ou lidando com a mudança; e (c) implica em apoiar coletivamente uma iniciativa de mudança planejada, ao invés de ser um esforço individual (Bordia et al., 2011; Oreg et al., 2011; Vakola et al., 2013, Vakola, 2016).

\section{Medidas sobre respostas comportamentais à mudança organizacional}

Para elencar as medidas utilizadas para aferir as respostas comportamentais positivas à mudança organizacional, foram revisadas as pesquisas nos últimos 15 anos. Foram identificados poucos instrumentos que aferem as respostas comportamentais positivas à mudança, pois a grande maioria dos estudos se dedica a respostas, tais como comprometimento com a mudança, que possui um caráter atitudinal (Kim et al., 2011).

Em 2011, Kim et al. avançaram ao desenvolver uma escala que buscava abranger comportamentos de apoio à mudança organizacional. A escala de Comportamento de Apoio à Mudança composta por três itens, eram avaliados em uma escala tipo Likert variando entre 1 (not at all) e 5 (to a very great extent). Os itens eram relacionados às contribuições feitas ao conselho representativo da organização, se a mudança havia sido discutida com colegas e se os problemas foram direcionados ao conselho representativo. A escala obteve consistência interna de 0,85 (T1) e 0,91 (T2).

\section{Resistência à mudança organizacional e as principais medidas do construto}

A expressão "resistência à mudança" foi utilizada pela primeira vez pelo psicólogo Kurt Lewin, utilizando uma metáfora das ciências físicas (Bortolotti, 2010). Desde então, a resistência é um tema de destaque por ser um fator crítico, sendo elencada como uma das principais barreiras ao sucesso das transformações organizacionais (Neiva \& Paz, 2012; Piderit, 2000), porque sua ocorrência provoca atrasos na implantação, demanda custos e gera instabilidades inesperadas no processo de mudança, ineficiência imprevista, procrastinação e esforços objetivando sabotar o processo de mudança (Franco, Neiva, Nery, \& Demo, 2016).

A maioria dos artigos publicados sobre resistência à mudança fornece suas próprias definições conceituais (Bouckenooghe, 2010) que, embora sejam diferentes, a maioria das definições considera o componente intencional/comportamental como uma força contrária que impede o sucesso da implantação da mudança e promove manutenção do status quo. Além disso, muitos estudos consideram tal fenômeno como um atributo individual disposicional (Nery \& Neiva, 2015).

Embora indesejada, a literatura indica que a resistência é a reação mais frequente à mudança organizacional (Piderit, 2000; Bouckenooghe, 2010) e, portanto, deve ser compreendida como elemento inerente às transformações cognitivas que ocorrem durante a mudança (George \& Jones, 2001).

Em 2003, Oreg validou uma escala para avaliar diretamente o componente disposicional que contribui para a resistência individual à mudança, denominada Resistance to Change Scale (RTC). A escala continha 44 itens e uma escala de concordância tipo Likert de 6 pontos. A validação indicou quatro fatores: rotina, reação emocional à mudança imposta, rigidez cognitiva e foco no curto prazo.

Em 2010, Bortolotti criou uma medida de resistência à mudança (RAM) com apoio na Teoria da Resposta ao Item (TRI), utilizando variáveis de causa, variáveis 
individuais, variáveis de contexto e resultado. A escala unidimensional contém 52 itens, e obteve 0,75 como alfa de Cronbach. Foi adotado o modelo de desdobramento, pela precisão em estimar o nível do respondente no traço latente resistência à mudança.

"A resistência à mudança é um traço latente ou uma variável latente" com essa afirmação Bortolotti (2010 p. 28) ressalta que suas características não podem ser medidas diretamente. Por isso, para elaboração da dimensão Resistência à Mudança Organizacional da escala de Respostas Comportamentais à Mudança foi adotada a seguinte definição: a emissão de comportamentos de oposição declarados ou não ao processo de mudança (Kim et al. 2011).

Ao adotar uma metodologia de pesquisa quantitativa, deve-se ter especial rigor ao escolher medidas que tenham sido validadas em estudos anteriores. Embora as escalas utilizadas neste estudo tenham sido validadas na população brasileira, faz-se necessário verificar se a estrutura fatorial de um instrumento já validado é replicável quando aplicados em outras amostras (Damásio, 2012).

Isso porque, segundo Huck (2009), há uma falsa compreensão a respeito da validade e confiabilidade das escalas. Embora uma escala obtenha bons índices de validade e confiabilidade para uma amostra, essa mesma escala pode ter sua estrutura fatorial modificada quando aplicada em outra, por exemplo, itens podem ser excluídos, reduzindo-se o instrumento ou podem-se alterar os índices de confiabilidade. Assim, é extremamente importante verificar a estrutura fatorial da medida utilizada, ainda que um instrumento tenha sido validado em outro estudo (Huck, 2009).

As respostas dos indivíduos à mudança organizacional envolvem reações emitidas pelos indivíduos durante os programas de mudança e apresentam valência positiva, negativa ou ambivalente (Piderit, 2000). Apesar da sua relevância, ainda existem poucas medidas validadas para o Brasil para acessar as respostas comportamentais à mudança (Bortolotti, 2010), e nenhuma aborda as duas dimensões: Comportamento de Apoio e Resistência. A mudança organizacional pode gerar diferentes emoções e reações que variam do otimismo ao medo, podendo incluir ansiedade, resistência, entusiasmo, incapacidade, motivação e pessimismo (Bortolotti, 2010).

Além disso, há uma ênfase grande às reações negativas à mudança organizacional, evidenciadas pela grande quantidade de estudos sobre resistência à mudança organizacional (Kim et al., 2011). Poucos estudos se dedicam a avaliar as respostas positivas aos programas de mudança organizacional, tais como comportamentos de apoio e comprometimento com a mudança (Kim et al., 2011), bem como poucos consideram esse rol de respostas e/ ou reações como componentes de um mesmo espectro de fenômeno apoio-resistência (Lines, 2005; Piderit, 2000). De maneira geral, o fenômeno pode envolver um espectro de reações positivas e negativas que podem ser estruturadas em um contínuo que se inicia no nível do indivíduo e se espalha para uma resposta coletiva, configurando-se em um padrão comportamental do grupo (Nery \& Neiva, 2015).

\section{Os comportamentos de apoio e resistência e o nível de análise}

O fenômeno das reações comportamentais a mudança tem sido analisado frequentemente no nível individual (Choi, 2011), contudo esse fenômeno também pode apresentar o nível coletivo sendo caracterizado por padrões comportamentais apresentados por um grupo de indivíduos (Bouckenooghe, 2010; Choi, 2011; George \& Jones, 2001), muitas vezes resultante do fenômeno de inércia organizacional. Nesse sentido, a inércia se manifestaria ou seria originária no nível individual e grupal como resistência (George \& Jones, 2001).

Fenômenos originários no nível individual de análise podem gerar atributos do nível macro ou meso (fenômeno da emersão, de emergir) a partir de diferentes processos de composição ou estruturação de atributos (Klein \& Kozlowski, 2000). Quando as pessoas são colocadas para trabalhar sob a influência dos mesmos eventos, elas podem desenvolver, com o passar do tempo, percepções compartilhadas sobre fenômenos do seu entorno organizacional. Embora a interpretação seja um processo cognitivo individual, ela é socialmente construída com base em marcos de referência social (interpretações coletivas). Além disso, os indivíduos podem desenvolver padrões comportamentais associados a essas interpretações da realidade, por meio de mecanismos sociais, muitas vezes chamados de contágio (Wiltermuth \& Heath, 2009).

Os comportamentos de apoio e resistência a mudanças surgem no nível individual, mas podem se tornar coletivos como padrões comportamentais que a maioria (talvez todos) dos membros organizacionais manifestam em virtude dos eventos que acontecem na organização (George \& Jones, 2001; Nery \& Neiva, 2015). Apesar do processo de emersão ocorrer, um dos meios de acesso ao fenômeno ainda é pela avaliação individual (Hox, 2010).

Chan (1998) defende a compreensão da natureza teórica do fenômeno em análise, e, a partir dela, o pesquisador pode adotar medidas que capturem o processo de emersão a partir do qual esse fenômeno se constrói. Para a construção de escalas baseadas em relato, a proposta de mudança de referente (Chan, 1998) possui grandes implicações. Nesse caso, o pesquisador entende que o construto do seu interesse surge a partir de processos ocorridos no nível inferior, mas a emersão implica na mudança do aspecto focado, embora permaneça com natureza semelhante, isto é, o fenômeno mantém a mesma natureza nos níveis micro e macro (ou meso), mas há mudança no foco de interesse. Quando ocorre a emersão, por mecanismos sociais de imitação ou contágio, os indivíduos manifestam padrões comportamentais de resistência, o que requer que a avaliação, com uso do 
referente coletivo, consiga detectar esse fenômeno coletivo. Assim, houve uma mudança do aspecto referido na avaliação, o que requer que a escala se diferencie em termos de referente. Esse propósito foi realizado na construção das duas versões da presente escala, na medida em que o nível de análise tem sido algo que preocupa os pesquisadores (Abbad, Puente-Palacios, \& Gondim, 2014) por suas consequências para a mensuração do construto.

Em resumo, as respostas dos indivíduos à mudança organizacional envolvem reações emitidas pelos indivíduos durante os programas de mudança e apresentam valência positiva, negativa ou ambivalente (Piderit, 2000). Apesar da sua relevância, ainda existem poucas medidas validadas para o Brasil para acessar as respostas comportamentais à mudança (Bortolotti, 2010), e nenhuma aborda as duas dimensões: Comportamento de Apoio e Resistência. A mudança organizacional pode gerar diferentes emoções e reações que variam do otimismo ao medo, podendo incluir ansiedade, resistência, entusiasmo, incapacidade, motivação e pessimismo (Bortolotti, 2010).

Além disso, há uma ênfase grande às reações negativas a mudança organizacional, evidenciadas pela grande quantidade de estudos sobre resistência a mudança organizacional (Kim et al., 2011). Poucos estudos se dedicam a avaliar as respostas positivas aos programas de mudança organizacional, tais como comportamentos de apoio e comprometimento com a mudança (Kim et al., 2011), bem como poucos consideram esse rol de respostas e/ou reações como componentes de um mesmo espectro de fenômeno apoio-resistência (Lines, 2005; Piderit, 2000).

De maneira geral, o fenômeno pode envolver um espectro de reações positivas e negativas que podem ser estruturadas em um contínuo que se inicia no nível do indivíduo e se espalha para uma resposta coletiva, configurando-se em um padrão comportamental do grupo (Nery \& Neiva, 2015).

Considerando o exposto, o objetivo deste estudo é descrever a estrutura fatorial e os indícios de validade psicométrica de construto da escala de respostas comportamentais à mudança organizacional para as amostras desta pesquisa. Como o nível de análise tem sido algo que preocupa os pesquisadores (Abbad et al., 2014), foram construídas duas versões com referente individual ou coletivo. A primeira (referente individual) avalia relatos de comportamentos realizados pelos indivíduos durante os processos de mudanças. A segunda (referente coletivo) avalia relatos de padrões de comportamentos coletivos percebidos pelos indivíduos durante os processos de mudanças.

\section{Método}

\section{Processo de construção da escala em suas duas versões}

A construção da escala se iniciou com a revisão de literatura e, em seguida, a operacionalização das definições e construção dos itens do instrumento. Tais definições se pautaram principalmente no trabalho teórico de Ashforth e Mael (1998). Posteriormente, cinco juízes especialistas em mudança organizacional classificaram os itens de acordo com a categoria a que se referiam e realizaram comentários sobre a redação dos itens. Com a devolução dos materiais, calculou-se o índice de concordância dos juízes em relação à classificação realizada previamente. Foram obtidos resultados correspondentes a $83 \%$ e $87 \%$. A partir das considerações feitas pelos juízes, alguns itens tiveram a redação ajustada ou foram retirados. Durante o processo de análise de juízes foi discutida a questão do referente da escala (individual ou coletivo). Para efeito das evidências de validade, foram construídas duas versões: uma com referente individual (eu) e outra com referente coletivo (os empregados, os colegas de trabalho, etc.)

Após a validação dos juízes, foi realizada a validação semântica com 60 trabalhadores de várias organizações que passavam por processos de mudança. Eles foram divididos em dois grupos que responderam às duas versões da escala. Cada um dos itens foi lido e foi discutida sua clareza. A partir dos comentários, ocorreram ajustes na redação de itens, nas instruções, e foram retirados itens com interpretações ambíguas. Após a avaliação semânti$\mathrm{ca}$, a escala, em suas duas versões foram aplicadas a empregados de organizações brasileiras que passavam por processos de mudança.

\section{Participantes}

Para a avaliação dos indícios de validade da escala com o referente coletivo, foi realizada uma pesquisa em uma empresa pública do Setor Elétrico, presente em 11 estados brasileiros das regiões Norte, Nordeste e CentroOeste. O convite para participar da pesquisa foi enviado para 2000 empregados da organização, sendo que 733 responderam ao questionário, o que totaliza uma taxa de retorno de 36,7\% com respondentes de 10 estados. Os dados estão na Tabela 1.

Tabela 1

Perfil dos Participantes da Pesquisa

\begin{tabular}{lcccc}
\hline \multirow{2}{*}{ Variável } & \multicolumn{2}{c}{ AFE } & \multicolumn{2}{c}{ AFC } \\
\cline { 2 - 5 } & $f$ & $\%$ & $f$ & $\%$ \\
\hline Sexo & 53 & 20,6 & 113 & 23,7 \\
$\quad$ Feminino & 197 & 76,7 & 358 & 75,2 \\
Masculino & 7 & 2,7 & 5 & 1,1 \\
$\quad$ Não informado & & & & \\
Escolaridade & & & 3 & 0,6 \\
$\quad$ Doutorado & 18 & 7,0 & 24 & 5,0 \\
Mestrado & 51 & 19,8 & 84 & 17,6 \\
Pós-graduação & 93 & 36,2 & 180 & 37,8 \\
Nível superior completo & 67 & 26,1 & 146 & 30,7 \\
Nível médio & 3 & 1,2 & 5 & 1,1 \\
Nível fundamental & 25 & 9,7 & 34 & 7,1 \\
$\quad$ Não informado & \multicolumn{4}{c}{$\mathrm{N}=257$} \\
\hline
\end{tabular}


A amostra que respondeu a versão com referente individual foi composta por membros de três organizações. A Organização 1 trata-se de uma empresa da área de financiamento bancário pública, possui 800 empregados e 437 foram convidados a participar da pesquisa, pois pertencem a mesma regional. Destes, somente 181 empregados responderam à pesquisa, taxa de retorno de $42 \%$. A Organização 2 é uma multinacional da área de vendas de produtos e serviços tecnológicos, possui 231 empregados e todos foram convidados a participar da pesquisa. Destes, 143 empregados responderam à pesquisa, taxa de retorno de $62 \%$. A Organização 3 é um banco privado com agências em todo o Brasil, contudo os questionários foram enviados para 1562 empregados das agências do Distrito Federal. Destes, 394 responderam, o que configura uma taxa de retorno de $27,14 \%$. Esses 718 respondentes foram divididos em duas amostras para realização da análise fatorial exploratória e confirmatória.

A idade média dos participantes (amostra com referente individual) foi de 26 anos $(D P=0,76)$, tendo $56 \%$ destes com idades entre 25 a 34 anos, $23 \%$ de 18 a 24 anos e $17 \%$ de 35 a 44 anos. A maioria eram mulheres, representando $57 \%$. Os empregados em sua maioria possuem tempo de empresa entre 6 meses a 2 anos $(36 \%)$ e de 2 anos a 5 anos (32\%). Quanto aos cargos que ocupam, $41,2 \%$ na área de vendas e operação; $29,5 \%$ trabalham em áreas-fins das organizações e 29,3\% em outras áreas dentro das organizações.

\section{Instrumento}

A escala de respostas comportamentais à mudança organizacional foi desenvolvida com 19 itens $\left(R^{2}=58,46 \%\right)$, a partir de uma estrutura teórica hipotetizada com dois fatores latentes. O instrumento possuía uma escala de concordância tipo Likert de 11 pontos (0 - discordo totalmente a 10 - concordo totalmente). A escala foi construída considerando os fatores que retratam reações comportamentais de apoio e resistência à mudança que têm sido estudadas pela literatura (Choi, 2011; Kim et al., 2011). A Tabela 2 apresenta as principais características do instrumento.

Tabela 2

Informações Gerais sobre a Escala*

\begin{tabular}{ccl}
\hline Construtos & Variáveis & \multicolumn{1}{c}{ Itens } \\
\hline $\begin{array}{c}\text { Respostas } \\
\text { Comportamentais }\end{array}$ & Apoio à Mudança & $\begin{array}{l}\text { Nove itens que avaliam a emissão de comportamentos que sejam dese- } \\
\text { jados pelo processo de mudanças, tais como flexibilidade, abertura ao } \\
\text { processo, aceitação, defensa da mudança }(\alpha=0,91) .\end{array}$ \\
& Resistência & $\begin{array}{l}\text { Dez itens que avaliam a emissão dos comportamentos de oposição ao } \\
\text { processo de mudança }(\alpha=0,90) .\end{array}$ \\
\hline
\end{tabular}

Nota. *As duas versões seguem a mesma composição e redação dos itens, somente há diferença no referente do item (eu ou os trabalhadores desta organização)

\section{Procedimentos de coleta de dados}

Os dados foram coletados por meio do software de pesquisa on-line Lime Survey. Na coleta de dados, o instrumento de pesquisa foi encaminhado aos sujeitos, por meio de correio eletrônico, que continha um texto explicativo, o endereço on-line da pesquisa, a senha para acesso ao questionário e o link para responder ao questionário.

Antes de acessar a pesquisa, os respondentes tiveram acesso ao Termo de Consentimento Livre e Esclarecido - TCLE, no qual constava um texto breve que informava o objetivo da pesquisa e solicitava o consentimento do participante.

Os dados passaram por avaliação de casos omissos, casos extremos uni e multivariados. Os casos extremos univariados, analisados por valores padronizados acima de 2,5 desvios foram mantidos quando inferiores a $5 \%$ em cada variável (Tabachnick \& Fidell, 2013). Os casos extremos multivariados foram analisados pela distância Mahalanobis $\left(\mathrm{D}^{2}\right)$ (Tabachnick \& Fidell, 2013). A avaliação dos pressupostos de normalidade foi realizada por meio dos testes Kolmogorov-Smirnov (K-S) e pelo teste Mardia (1971).

\section{Procedimento de análise dos dados}

Inicialmente, as escalas foram submetidas a uma inspeção inicial realizada por meio da análise dos componentes principais (Principal Components - PC) e também por meio da análise fatorial (Principal Axis Factoring), conforme indicações de Pasquali (2010) e Damásio (2012). Como se tratava de uma inspeção inicial e como Damásio (2012) afirma que os resultados da PC são mais instáveis e podem gerar resultados ambíguos, os resultados dessa inspeção inicial foram comparados com o objetivo de estimar o número de fatores iniciais a serem extraídos, além de verificar as relações lineares entre as variáveis e a fatorabilidade da matriz de correlações. Esse procedimento foi usado somente para realizar uma primeira análise da escala. A análise fatorial exploratória propriamente dita foi realizada pela extração dos eixos principais, conforme pontua Damásio (2012). Para obter as estruturas fatoriais, utilizou-se o método de fatoração dos eixos principais 
(Principal Axis Factoring - PAF) com rotação oblíqua promax. Os critérios utilizados para a decisão em relação à retenção de fatores foram: variância explicada pelo fator acima de $3 \%$; autovalores superiores a 1 ; formato do scree plot; a análise paralela de Horn e a consistência teórica (Damásio, 2012; Pasquali, 2010).

A inspeção da matriz de correlações foi verificada por meio do tamanho das correlações. Também foi observado o teste de adequação da amostra de KaiserMeyer-Olkin (KMO) em que se esperam valores próximos a 1,00 , e o determinante da matriz, que deve ter um valor baixo, sugerindo que as variáveis são intercorrelacionadas (Damásio, 2012). Após a extração dos fatores foram analisadas a interpretabilidade coerente com a abordagem teórica em estudo (Pasquali, 2010), e a consistência dos fatores, realizada por meio do alfa de Cronbach (Pasquali, 2010). Para estimação e extração de fatores, foram considerados o método Principal Axis Factoring e os métodos Unweighted Least Squares (ULS) e Weighted Least Squares Mean and Variance Adjusted (WLSMV), por se tratar de medida que usa a escala Likert (de acordo com recomendações de Asún, RdzNavarro \& Alvarado, 2015).

A escala de respostas comportamentais à mudança organizacional também foi submetida à verificação da estrutura fatorial pela realização da análise fatorial confirmatória via equações estruturais. Conforme relatado, foram analisados previamente os pressupostos de normalidade, linearidade e homogeneidade de variâncias, além de problemas em relação à multicolinearidade e à singularidade. Considerou-se, em todas as análises, a matriz de covariâncias e adotou-se como método de estimação o da máxima verossimilhança (Maximum Likelihood).

Os modelos foram avaliados por meio dos seguintes índices: razão entre qui-quadrado $\left(\chi^{2}\right)$ e graus de liberdade (gl), NFI (Normed Fit Index), TLI (Tucker-Lewis Index), CFI (Comparative Fit Index), GFI (Godness-of-Fit Index), AGFI (Adjusted Godness-of-Fit Index) com valores ideais próximos ou acima de 0,90 e RMSEA (Root Mean Square Error of Aproximation), com valor ideal próximo ou inferior a 0,08 e intervalo de confiança de $90 \%$ (IC90\%) (Hox, 2010). Para realizar as reespecificações nos modelos, seguiram-se os seguintes critérios, sugeridos por Byrne (2016): (a) apenas se alteraram/eliminaram as trajetórias e/ou erros correlacionados quando o índice de modificação era superior a $50(p<0,001)$; (b) a exclusão do item era justificável de um ponto de vista teórico; (c) sempre que possível, optou-se pela eliminação de um determinado item do par ao invés de se acrescentar a correlação entre os erros; (d) optou-se pela eliminação de itens cujos erros apresentavam covariância elevada com dois ou mais erros de outros itens. Avaliou-se, ainda, a matriz de resíduos normalizados. De acordo com Hox (2010), no máximo 5\% dos resíduos podem estar fora do intervalo $[-2,58 ; 2,58]$, o que implica nível de significância de 0,05 .

\section{Resultados}

\section{Análise da estrutura fatorial da Escala de Respostas Comportamentais à Mudança Organizacional - Versão com Referente Individual}

A estrutura fatorial da escala de comportamentos de apoio e resistência à mudança organizacional foi verificada por meio de análises fatorial exploratória e confirmatória, utilizando uma amostra 718 respondentes. Os questionários incompletos foram excluídos; foram mantidos os casos extremos univariados, pois estes eram inferiores a $5 \%$ em cada variável (Tabachnick \& Fidell, 2013). Identificaram-se casos extremos multivariados $\left(\mathrm{D}^{2} \geq=43,820 ; d f=19 ; p<0,001\right)$ que foram excluídos. Para a realização da análise fatorial exploratória, foi feita uma seleção aleatória de 294 casos e a análise fatorial confirmatória foi realizada com uma seleção aleatória de 424 casos. Nenhuma variável foi submetida a transformação, pois não foram detectados desvios de normalidade multivariada (Mardia, 1971).

Para analisar a fatorabilidade dos dados e estimar o número de fatores, os 19 itens da escala de respostas comportamentais à mudança organizacional foram submetidos à análise dos componentes principais e a análise dos eixos principais em virtude dos resultados instáveis produzidos pela PC (Damásio, 2012). Os resultados dessa inspeção foram muito similares nos dois procedimentos. O valor do KMO obtido foi para a amostra com referente individual foi de $0,827(p<0,001)$. Foi solicitado itens com valor mínimo de carga de 0,45 . A matriz de correlações indicou a presença de relações lineares entre as variáveis, a ausência de multicolinearidade e de singularidade. Observaram-se $50 \%$ dos valores de correlação superiores a 0,30 .

A inspeção inicial sugeriu uma estrutura empírica com dois fatores que explicou $48,7 \%$ da variância total das respostas dos participantes aos itens do questionário na versão referente individual. A análise do gráfico de sedimentação (Figura 1) sugere a presença de três fatores. A pertinência teórica indica a presença de dois fatores. A análise paralela de Horn e a consistência teórica dos fatores (Damásio, 2012; Pasquali, 2010) indicaram dois fatores a serem retidos.

Após a escolha da extração de dois fatores, para estimação e extração de fatores, foram considerados o método Principal Axis Factoring e os métodos Unweighted Least Squares (ULS) e Weighted Least Squares Mean and Variance adjusted (WLSMV), por se tratar de medida que usa a escala Likert (de acordo com recomendações de Asún et al., 2015). Abaixo estão apresentados os resultados da extração pelo método Unweighted Least Squares (ULS).

Os valores das cargas fatoriais e da variância explicada estão apresentados na Tabela 3. 


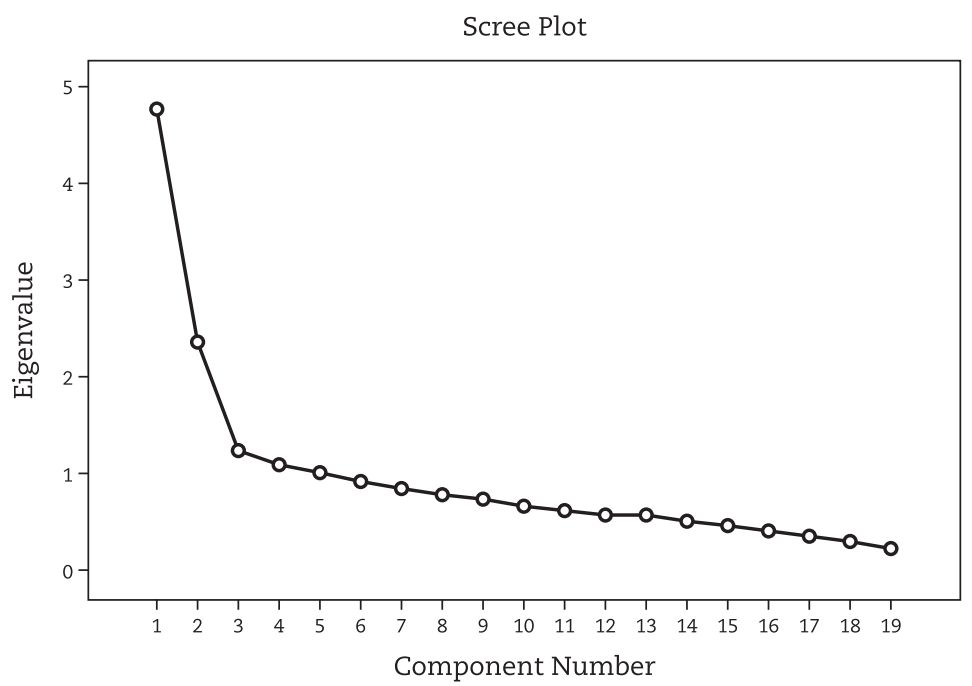

Figura 1. Gráfico de sedimentação da Escala (referente individual)

Tabela 3

Resultados da Escala Versão Referente Individual

\begin{tabular}{|c|c|c|c|}
\hline \multirow{2}{*}{ Itens } & \multicolumn{2}{|c|}{ Cargas fatoriais } & \multirow{2}{*}{$\mathrm{h}^{2}$} \\
\hline & Fator 1 & Fator 2 & \\
\hline Eu ajo de acordo com as novas diretrizes. & 0,453 & & 0,562 \\
\hline Eu critico os responsáveis que trabalham com o processo de mudança. & & & 0,667 \\
\hline Eu declaro que sou a favor das mudanças ocorridas. & 0,533 & & 0,494 \\
\hline Eu boicoto o processo de mudança. & & 0,523 & 0,572 \\
\hline Eu busco informações sobre a mudança. & 0,578 & & 0,610 \\
\hline Eu adio as ações do processo de mudança. & & 0,531 & 0,433 \\
\hline Eu me comporto de maneira favorável à mudança. & 0,755 & & 0,671 \\
\hline Eu ridicularizo os responsáveis pela mudança. & & 0,595 & 0,489 \\
\hline Eu escondo informações relevantes para o processo de mudança. & & 0,524 & 0,449 \\
\hline Eu participo ativamente do processo de mudança. & & & 0,446 \\
\hline Eu não percebo que a mudança esteja acontecendo. & & 0,503 & 0,567 \\
\hline Eu adoto os novos comportamentos necessários à mudança. & 0,662 & & 0,643 \\
\hline Eu apresento críticas negativas à mudança. & & 0,489 & 0,608 \\
\hline Eu realizo ações que facilitam o processo de mudança. & 0,684 & & 0,583 \\
\hline Eu desobedeço às novas regras implantadas pela mudança. & & & 0,420 \\
\hline Eu defendo a mudança para os colegas. & 0,731 & & 0,603 \\
\hline Eu finjo que concordo com o processo de mudança. & & 0,590 & 0,666 \\
\hline Eu participo das decisões da empresa sobre a mudança. & & & 0,426 \\
\hline Eu faço piadas sobre o processo de mudança. & & 0,655 & 0,571 \\
\hline $\mathrm{N}$ & 294 & & \\
\hline Número de itens & 7 & 8 & \\
\hline Eigenvalue & 4,741 & 2,356 & \\
\hline Percentual de variância & 36,30 & 12,40 & \\
\hline Alfa de Cronbach & 0,81 & 0,79 & \\
\hline
\end{tabular}

As correlações item-total foram moderadas, sugerindo coerência entre os itens e o fator, nas duas versões da escala. Os itens 2, 10, 15 e 18 não obtiveram valor de carga fatorial superior a 0,45 , por isso, foram excluídos da escala. A validação da escala foi fundamentada na teoria do construto, portanto, apesar do gráfico de declividade e a análise dos autovalores indicarem três fatores, foram adotados dois fatores devido à pertinência teórica. 
A análise fatorial confirmatória foi realizada com uma subamostra de $41 \%$ respondentes do banco de dados pesquisa $(N=294)$. A correlação entre fatores foi de 0,32 , o que corrobora a opção por uma estrutura bifatorial.

Após a escolha da extração de dois fatores, para estimação e extração de fatores, foram considerados o método Principal Axis Factoring e os métodos Unweighted Least Squares (ULS) e Weighted Least Squares Mean and Variance adjusted (WLSMV), por se tratar de medida que usa a escala Likert (de acordo com recomendações de Asún et al., 2015). Abaixo estão apresentados os resultados da extração pelo método Unweighted Least Squares (ULS).

Para realização da análise fatorial confirmatória, foram incluídos no modelo apenas os itens com os maiores valores de carga acima de 0,45 . Observou-se que os itens do instrumento possuem um padrão assimétrico. Os valores de assimetria variaram de $-0,07$ até 0,21 . Os valores de curtoses foram reduzidos e significativos, variando de $-0,05$ até 0,34 . O coeficiente de normalidade multivariada de Mardia (1971) também sugere um padrão moderadamente não normal da distribuição dos dados. Considerando-se que esse padrão não foi elevado, optou-se pela utilização do algoritmo da máxima verossimilhança para estimação dos parâmetros de acordo com a utilização de outros autores (Byrne, 2016). A Tabela 4 apresenta os resultados encontrados na amostra composta por 424 casos válidos. No caso dessa versão com referente no nível individual, os alfas de Cronbach extraídos a partir dos dados das duas amostras (exploratória e confirmatória) foram muito próximos (amostra $\mathrm{AFE}=0,81 \mathrm{e}$ $0,79$; amostra $\mathrm{AFC}=0,85$ e 0,80$)$.

Conforme pode ser observado na Tabela 4, o modelo inicial obteve indicadores aceitáveis. Analisando os resultados das análises post hoc, foi observada uma covariância significativa entre erros, não prevista no modelo inicial. Após a reespecificação do modelo, houve uma melhora sensível nos índices de ajuste, em relação aos obtidos no modelo inicial. Os itens que possuem covariância entre si guardam semelhança de conteúdo, portanto a reespecificação do modelo respeitou recomendações de justificativa teórica para sua adoção. A Figura 2 apresenta o modelo reespecificado com as covariâncias estimadas.

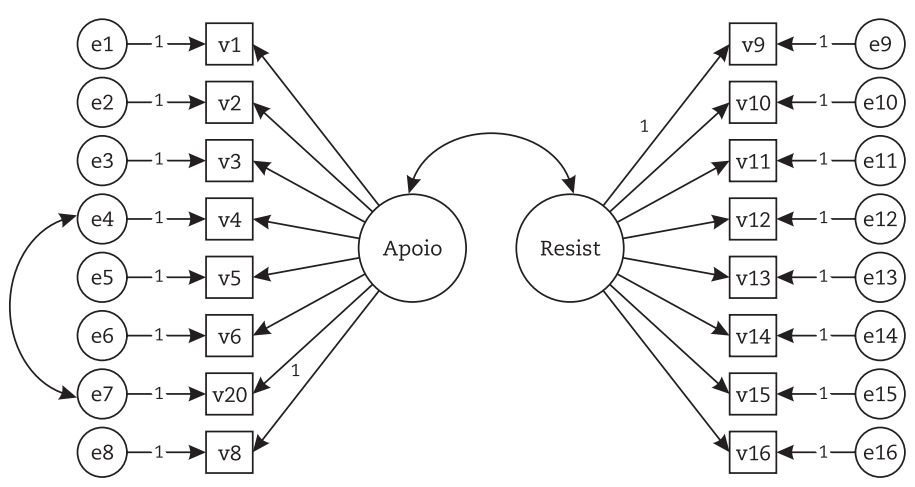

Figura 2. Modelo reespecificado da escala com coeficientes (referente individual)

Tabela 4

Índices de Adequação dos Modelos da Escala com Eferente Individual

\begin{tabular}{|c|c|c|c|c|c|c|c|c|c|c|c|}
\hline & \multicolumn{11}{|c|}{ Modelo Bifatorial } \\
\hline & $\chi^{\prime \prime}$ & gl & $\chi^{\prime \prime} \gamma . \lambda$. & NFI & TLI & CFI & GFI & AGFI & RMSEA (IC 95\%) & $\Delta \chi^{2 *}$ & $\Delta g l$ \\
\hline Modelo inicial & 243,26 & 103 & 2,36 & 0,91 & 0,90 & 0,90 & 0,91 & 0,89 & $0,06(0,05-0,08)$ & & \\
\hline Modelo reespecificado & 182,35 & 88 & 2,07 & 0,94 & 0,93 & 0,93 & 0,95 & 0,92 & $0,05(0,03-0,06)$ & 60,91 & 15 \\
\hline
\end{tabular}

Nota. ${ }^{*} p<0,001 ; g l=$ graus de liberdade; IC=intervalo de confiança $(95 \%) ; \Delta \chi^{2}=$ diferença de qui-quadrado; $\Delta g l=$ diferença de graus de liberdade

Neste estudo, os índices de ajuste encontrados na análise fatorial confirmatória para a escala de Respostas Comportamentais à Mudança Organizacional foram consideradas aceitáveis $\left[\chi^{2}(88, N=424)=182,35\right.$, $p<0,05 ; \chi^{2} / g l=2,07 ; \mathrm{NFI}=0,94 ; \mathrm{TLI}=0,93 ; \mathrm{CFI}=0,93$; GFI $=0,95$; AGFI 0,92 ; RMSEA (IC) $=0,05(0,03-0,06)]$. Para essa solução fatorial, todos os parâmetros estimados foram estatisticamente significativos. 


\section{Estrutura fatorial da Escala de Respostas Comportamentais à Mudança Organizacional - Versão com Referente no Coletivo}

A estrutura fatorial da escala de comportamentos de apoio e resistência à mudança organizacional, versão com referente coletivo, foi verificada por meio das análises fatorial exploratória e confirmatória, com 733 respondentes. Os questionários incompletos foram excluídos; foram mantidos os casos extremos univariados, pois estes eram inferiores a 5\% em cada variável (Tabachnick \& Fidell, 2013). Identificaram-se casos extremos multivariados $\left(\mathrm{D}^{2} \geq=43,820 ; d f=19 ; p<0,001\right)$ que foram excluídos. Para a realização da análise fatorial exploratória, foi feita uma seleção aleatória de 257 casos, e a análise fatorial confirmatória foi realizada com uma seleção aleatória de 476 casos. Nenhuma variável foi submetida a transformação, pois não foram detectados desvios de normalidade multivariada (Mardia, 1971).

Para analisar a fatorabilidade dos dados e estimar o número de fatores, os 19 itens da escala de respostas comportamentais à mudança organizacional foram submetidos à análise dos componentes principais e a análise dos eixos principais em virtude dos resultados instáveis produzidos pela PC (Damásio, 2012). Os resultados dessa inspeção foram muito similares nos dois procedimentos. A inspeção inicial da matriz indicou um KMO aceitável, cujo valor obtido foi $0,934(p<0,001)$. A matriz de correlações indicou a presença de relações lineares entre as variáveis, a ausência de multicolinearidade e de singularidade. Observaram-se 50\% dos valores de correlação superiores a 0,30 e determinante muito próximo de zero.

A análise da inspeção inicial sugeriu uma estrutura empírica com dois fatores que explicou 59,97\% da variância total das respostas dos participantes aos itens do questionário. A análise do gráfico de sedimentação (Figura 3) sugere a presença de três fatores. A pertinência teórica indica a presença de dois fatores. A análise paralela de Horn e a consistência teórica (Damásio, 2012; Pasquali, 2010) indicaram dois fatores a serem retidos.

Após a escolha da extração de dois fatores, para estimação e extração de fatores, foram considerados o método Principal Axis Factoring e os métodos Unweighted Least Squares (ULS) e Weighted Least Squares Mean and Variance adjusted (WLSMV), por se tratar de medida que usa a escala Likert (de acordo com recomendações de Asún et al., 2015). Abaixo estão apresentados os resultados da extração pelo método Unweighted Least Squares (ULS). Abaixo estão apresentados os resultados da extração pelo método Unweighted Least Squares (ULS). Os valores das cargas fatoriais e da variância explicada estão apresentados na Tabela 5 .

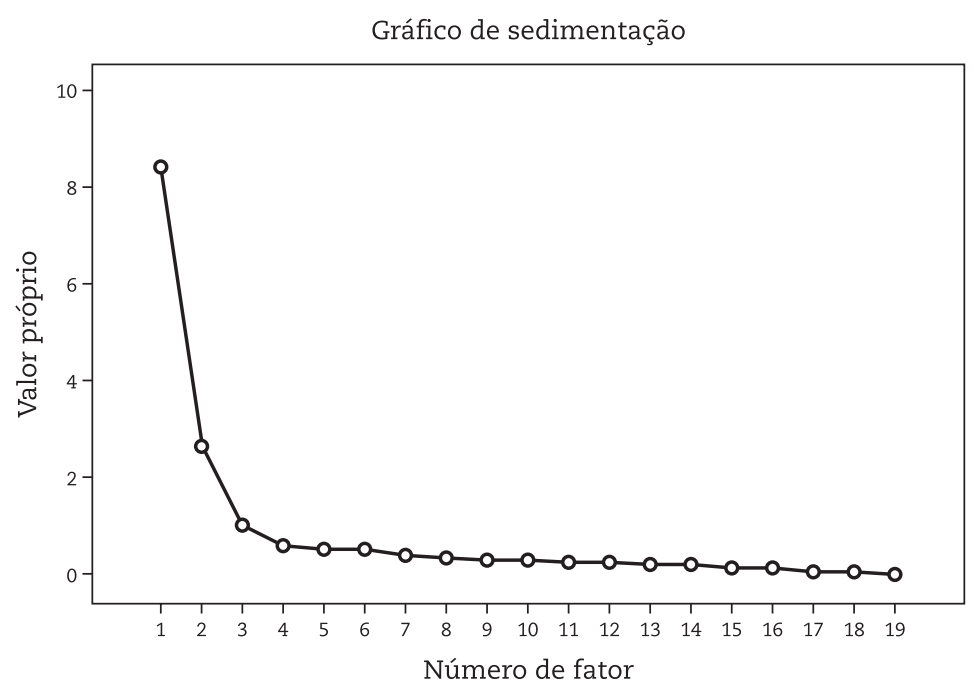

Figura 3. Gráfico de sedimentação da Escala de Respostas Comportamentais (Referente Coletivo).

Tabela 5

Resultados da Análise Fatorial Exploratória dos Itens da Escala (Referente Coletivo)

\begin{tabular}{lrr}
\multicolumn{1}{c}{ Itens } & Cargas Fatoriais & $\mathrm{h}^{2}$ \\
\cline { 2 - 3 } & Fator 1 & Fator 2 \\
\hline 1. Os colaboradores agem de acordo com as novas diretrizes. & 0,73 & 0,60 \\
2. Os colaboradores criticam os gerentes que trabalham com o processo de mudança. & 0,50 & 0,42 \\
3. Os colaboradores declaram ser a favor das mudanças ocorridas. & 0,73 & 0,67
\end{tabular}


Tabela 5 (continuação)

Resultados da Análise Fatorial Exploratória dos Itens da Escala (Referente Coletivo)

\begin{tabular}{|c|c|c|c|}
\hline \multirow{2}{*}{ Itens } & \multicolumn{2}{|c|}{ Cargas Fatoriais } & \multirow{2}{*}{$h^{2}$} \\
\hline & Fator 1 & Fator 2 & \\
\hline 4. Os colaboradores boicotam o processo de mudança. & & 0,76 & 0,66 \\
\hline 5. Os colaboradores buscam informações sobre a mudança. & 0,58 & & 0,39 \\
\hline 6. Os colaboradores adiam as ações do processo de mudança. & & 0,75 & 0,54 \\
\hline 7. Os colaboradores se comportam de maneira favorável à mudança. & 0,79 & & 0,74 \\
\hline 8. Os colaboradores ridicularizam os agentes de mudança. & & 0,76 & 0,71 \\
\hline 9. Os colaboradores escondem informações relevantes para o processo de mudança. & & 0,75 & 0,50 \\
\hline 10. Os colaboradores participam ativamente do processo de mudança. & 0,80 & & 0,72 \\
\hline 11. Os colaboradores negam que a mudança esteja acontecendo. & & 0,59 & 0,43 \\
\hline 12. Os colaboradores adotam os novos comportamentos necessários para mudança. & 0,86 & & 0,79 \\
\hline 13. Os colaboradores apresentam críticas negativas à mudança. & & & 0,42 \\
\hline 14. Os colaboradores realizam ações que facilitam o processo de mudança. & 0,84 & & 0,74 \\
\hline 15. Os colaboradores desobedecem às novas regras implantadas pela mudança. & & 0,73 & 0,52 \\
\hline 16. Os colaboradores defendem a mudança para os colegas. & 0,64 & & 0,71 \\
\hline 17. Os colaboradores fingem que concordam com o processo de mudança. & & & 0,64 \\
\hline 18. Os colaboradores participam das decisões da empresa sobre a mudança. & & & 0,29 \\
\hline 19. Os colaboradores fazem piada sobre o processo de mudança. & & & 0,62 \\
\hline$N$ & 239 & & \\
\hline $\mathrm{N}^{\circ}$ de itens & 8 & 7 & \\
\hline Eigenvalue & 8,73 & 2,53 & \\
\hline \% da Variância Explicada & 45,99 & 13,31 & \\
\hline Alfa de Cronbach & 0,93 & 0,881 & \\
\hline
\end{tabular}

As correlações item-total foram moderadas, sugerindo coerência entre os itens e o fator. Os itens 13, 1718 e 19 não obtiveram valor de carga fatorial superior a 0,50 , por isso, foram excluídos da escala. Os indícios de validade da escala foram fundamentados na teoria do construto. Apesar do gráfico de declividade e a análise dos autovalores indicarem três fatores, foram adotados dois fatores devido a pertinência teórica. A análise fatorial confirmatória foi realizada com uma subamostra de $30 \%$ respondentes do banco de dados pesquisa $(N=239)$.

Os alfas de Cronbach extraídos a partir dos dados das duas amostras (exploratória e confirmatória) foram muito próximos (Amostra $\mathrm{AFE}=0,93$ e 0,88; amostra $\mathrm{AFC}=0,93$ e 0,89$)$. A correlação entre fatores foi de 0,32 , o que justifica a opção por uma estrutura bifatorial.

Observou-se que os itens do instrumento possuem um padrão assimétrico. Os valores de assimetria variaram de $-0,06$ até 0,19 . Os valores de curtoses foram reduzidos e significativos, variando de $-0,05$ até 0,36 . O coeficiente de normalidade multivariada de Mardia (1971) também sugerem um padrão moderadamente não normal da distribuição dos dados. Considerando-se que esse padrão não foi elevado, optou-se pela utilização do algoritmo da máxima verossimilhança para estimação dos parâmetros de acordo com a utilização de outros autores (Byrne, 2016). A Tabela 6 apresenta os resultados encontrados na amostra composta por 400 casos válidos.

Tabela 6

Índices de Adequação dos Modelos Testados na AFC da Escala (Referente Coletivo)

\begin{tabular}{|c|c|c|c|c|c|c|c|c|c|c|c|}
\hline & \multicolumn{11}{|c|}{ Modelo Bifatorial } \\
\hline & $\chi^{\prime \prime}$ & $g l$ & $\chi^{\prime \prime} \gamma . \lambda$. & NFI & TLI & CFI & GFI & AGFI & RMSEA (IC 95\%) & $\Delta \chi^{2^{*}}$ & $\Delta g l$ \\
\hline Modelo Inicial & 130,96 & 43 & 3,04 & 0,94 & 0,95 & 0,96 & 0,94 & 0,91 & $0,07(0,05-0,08)$ & & \\
\hline Modelo Reespecificado & 86,91 & 42 & 2,06 & 0,96 & 0,97 & 0,98 & 0,96 & 0,94 & $0,05(0,03-0,06)$ & 44,05 & 1 \\
\hline
\end{tabular}

Nota. ${ }^{*} p<0,001 ;$ gl=graus de liberdade; IC=intervalo de confiança (95\%); $\Delta \chi^{2}=$ diferença de qui-quadrado; $\Delta g l=$ diferença de graus de liberdade 
Conforme pode ser observado na Tabela 6, o modelo inicial obteve indicadores aceitáveis. Analisando os resultados das análises post hoc, foi observada uma covariância significativa entre erros, não prevista no modelo inicial. Após a reespecificação do modelo houve uma melhora sensível nos índices de ajuste, em relação aos obtidos no modelo inicial. Os itens que possuem covariância entre si guardam semelhança de conteúdo, portanto a reespecificação do modelo respeitou recomendações de justificativa teórica para sua adoção. A Figura 4 apresenta o modelo reespecificado com as covariâncias estimadas.

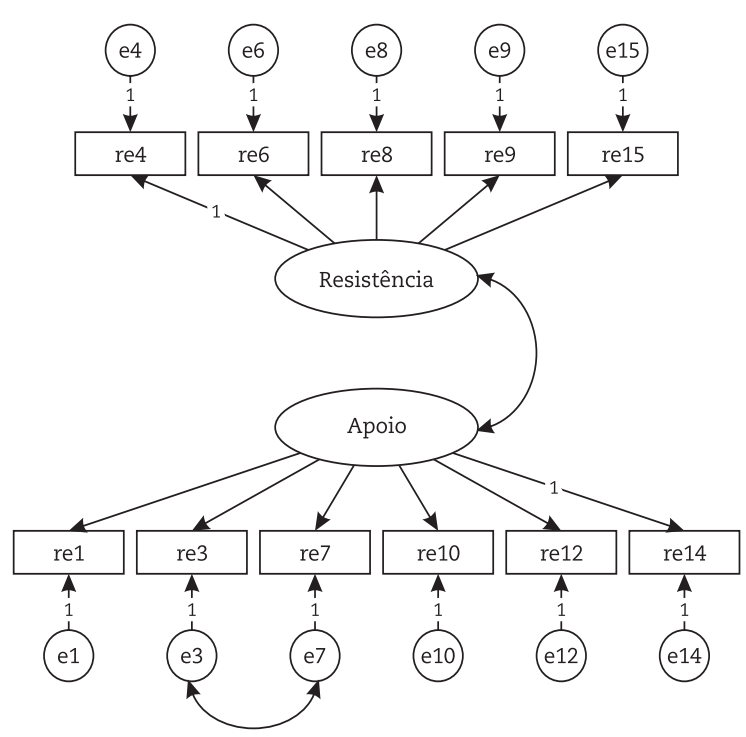

Figura 4. Modelo Reespecificado da Escala com Coeficientes (referente coletivo)

Neste estudo, os índices de ajuste encontrados na análise fatorial confirmatória para a escala de Respostas Comportamentais à Mudança Organizacional foram considerados aceitáveis $\left[\chi^{2}(42, N=400)=86,91, p<0,05 ; \chi^{2}\right]$ $g l=2,06$; NFI=0,96; TLI=0,97; CFI $=0,98 ; \mathrm{GFI}=0,96$; AGFI $=0,94$; RMSEA (IC) $=0,05(0,03-0,06)]$. Para essa solução fatorial, todos os parâmetros estimados foram estatisticamente significativos.

\section{Discussão e Conclusões}

O presente estudo é relevante, pois descreve a estrutura fatorial e aponta indícios de validade psicométrica da escala de respostas comportamentais à mudança organizacional para a pesquisa em questão. À vista disso, apresenta indícios de validade de uma escala generalista que pode ser aplicada em diversas organizações, facilitando a coleta de indicadores comportamentais relacionados ao sucesso da mudança, tanto no nível individual quanto coletivo, uma lacuna previamente indicada na literatura (Piderit, 2000; Oreg et al., 2011).

A escala demonstrou bons índices de validade e confiabilidade e adequação e é indicada para utilização em outras amostras, em pesquisas sobre mudança organizacional. Sua estrutura bifatorial - comportamentos de apoio e resistência - possui bons índices de consistência interna e validade de construto e dá suporte as proposições teóricas sobre resistência de Ashforth e Mael (1998).

No referente individual que avalia os comportamentos dos colaboradores durante os processos de mudanças, os resultados foram considerados aceitáveis e corroboram com modelos teóricos de respostas comportamentais às mudanças apresentados na literatura (Bortolotti, 2010; Hox, 2010; Peccei, Giangreco, \& Sebastiano, 2011), indicando variáveis individuais e situacionais como explicativas em $48,7 \%$ do processo. Sob esse aspecto, os indivíduos ao se depararem com uma mudança procuram "dar sentido" e significado ao processo (Lysova, Richardson, Shapova, \& Jansen, 2015).

Quanto ao referente coletivo, os resultados apontam um poder explicativo de $59,97 \%$ na direção de que a avaliação individual contribui para a formação de padrões comportamentais dos membros da organização e estes são influenciados pelos acontecimentos internos durante a mudança (George \& Jones, 2001; Nery \& Neiva, 2015). Nesse sentido, este estudo inovou ao incorporá-lo no estudo da área, concomitantemente ao estudar a polaridade das respostas às mudanças organizacionais- apoio-resistência, o que é ressaltado por muitos pesquisadores (Bordia et al., 2011; Oreg et al., 2011; Vakola et al., 2013, Vakola, 2016). 
A escala de respostas comportamentais à mudança organizacional diferencia-se de escalas anteriores devido a seu caráter integrador de duas dimensões comportamentais - Comportamento de Apoio e Resistência - na mesma escala. Esses aspectos são considerados críticos para o sucesso da mudança, mas em outras escalas presentes na literatura apresentam estrutura unidimensional, aferindo ou a resistência (Bortolotti, 2010; Oreg, 2003) ou os comportamentos de apoio à mudança organizacional (Kim et al., 2011). Considera-se que a estrutura fatorial com dois fatores está aderente à definição do construto e encontra fundamentação teórica, além de ser mais abrangente. As correlações entre os fatores estiveram em torno de 0,30 , o que reforça a opção por uma estrutura bifatorial (Damásio, 2012; Pasquali, 2010).

Existe uma discussão na literatura sobre a importância dos métodos para estimação e extração de fatores. No caso do presente estudo, para as duas versões do instrumento, foram considerados o método Principal Axis Factoring e os métodos Unweighted Least Squares (ULS) e Weighted Least Squares Mean and Variance adjusted (WLSMV), por se tratar de medida que usa a escala Likert (Asún et al., 2015). Os resultados apresentados se referem a extração pelo método Unweighted Least Squares (ULS), mas os métodos Principal Axis Factoring e Weighted Least Squares Mean and Variance adjusted apresentaram resultados similares aos apresentados neste artigo. Embora todos os procedimentos tenham mostrado capacidade de produzir soluções válidas e estáveis, ULS e WLSMV obtiveram um viés notavelmente mais baixo e em ambos os parâmetros, as estimativas foram robustas para os cenários mais difíceis: item com distribuição assimétrica, baixa qualidade do item e pequenos tamanhos de amostra. Essa realidade parece não ter se reproduzido no presente estudo. As estimativas maximum likelihood, além de outras, quando usadas em matrizes de correlação tetra ou policírica produzem resultados bastante semelhantes a outras, especialmente em grandes amostras (Asún et al., 2015), quando os pressupostos de normalidade são assegurados.

A comparação dos resultados nas duas versões (referente individual e coletivo) permitem inferir a estrutura extremamente semelhante dos fenômenos em diferentes níveis conforme aponta os trabalhos de emersão por composição (Klein \& Kozlowski, 2000; Chan, 1998; George \& Jones, 2001). Vale ressaltar que os estudos atuais buscam avaliar de forma isolada os comportamentos de apoio e de resistência as mudanças organizacionais e somente no nível individual.
Espera-se que este estudo contribua para aprofundar a temática relativa a comportamentos de apoio e resistência à mudança organizacional, reconhecendo variáveis individuais e coletivas que devem ser consideradas em estratégias de intervenção. Esta pesquisa apresentou em seus resultados a adequação das medidas e robustez, como apontado nas pesquisas internacionais, que evidenciam a necessidade de mensurar comportamento de apoio (Kim et al., 2011) e de resistência a mudanças organizacionais (Oreg,2003), embora a questão cultural tenha significante importância nos estudos de mudança organizacional (Oreg, et al., 2008).

Mesmo apresentando resultados promissores, indica-se para estudos futuros, a submissão de ajustes, por exemplo, em termos de redação de itens, ou mesmo a ampliação da escala com a inclusão de novas questões, visando a melhores ajustes e, consequentemente, maior acurácia da medida.

No âmbito acadêmico, há uma delimitação do construto, separação da sua análise em níveis, a escala apresenta bons índices de validade/confiabilidade, está adaptada ao contexto brasileiro e é rapidamente aplicada. Por suas características, essa escala possibilita a identificação dos principais antecedentes e consequentes das respostas comportamentais à mudança organizacional, tanto no nível individual quanto coletivo, possibilitando assim, a produção acadêmica e aprimorando as medidas sobre o fenômeno (Neiva \& Paz, 2012).

A contribuição prática é que a escala pode ser utilizada pelas organizações para gerenciar as iniciativas de mudança, por meio do diagnóstico de variáveis-chave para o sucesso da mudança organizacional, permitindo o monitoramento a fim de mitigar possíveis efeitos negativos e potencializar eventuais efeitos positivos, além de avaliar o quanto isso é compartilhado pelos indivíduos (Chan, 2008).

Apesar dos avanços metodológicos e práticos, este estudo possui limitações que precisam ser explicitadas. Uma delas é que a amostra desse estudo foi coletada em apenas uma organização pública. Outra questão importante é a necessidade de novas aplicações dessa escala, buscando verificar sua qualidade psicométrica em outras amostras (Damásio, 2012). Assim, reitera-se que ainda é possível aprimorar essa escala e seus índices de adequação. Para isso, sugere-se que os itens que apresentaram covariância sejam suprimidos e novos indicadores dos eventos mensurados por esses itens sejam formulados. Dessa forma, será possível melhorar a adequação do modelo.

\section{Referências}

Abbad, G. S., Puente-Palacios, K. E., \& Gondim, S. G. (2014). Abordagens metodológicas em psicologia organizacional e do trabalho. Revista Brasileira de Psicologia, 1(2), 71-88. 
Ashforth, B. E., \& Mael, F. A. (1998). The power of resistance: Sustaining valued identities. Em R. M. Kramer \& M. A. Neale editors. Power and influence in organizations. Thousand Oaks, California: Sage.

Asún, R. A., Rdz-Navarro, K., \& Alvarado, J. M. (2015). Developing multidimensional likert scales using item factor analysis: The case of four-point items. Sociological Methods E Research. doi: 10.1177/0049124114566716

Bordia, P. Restubog, S. L. D., Jimmieson, N., \& Irmer, B. (2011). Haunted by the Past: Effects of poor change management history on employee attitudes and turnover. Group \& Organization Management, 36(2) 191-222. doi:10.1177/1059601110392990

Bortolotti, S. L. V. (2010). Resistência à mudança organizacional: Medida de avaliação por meio da Teoria da Resposta ao Item. Universidade Federal de Santa Catarina, Florianópolis.

Bouckenooghe, D. (2010). Positioning change recipients' attitudes toward change in the organizational change literature. The Journal of Applied Behavioral Science, 46(4), 500-531. doi: 10.1177/0021886310367944

Byrne, B. M. (2016). Structural equation modeling with Amos: Basic concepts, applications, and programming (3rd ed.). New York, NY: Routledge Group.

Chan, D. (1998). Functional relations among constructs in the same content domain at different levels of analysis: A typology of compositions models. Journal of Applied Psychology, 83, 234-246. doi: 10.1037/0021-9010.83.2.234

Choi, M. (2011). Employees' attitudes toward organizational change: A literature review. Human Resource Management, 50(4), 479-500. doi: $10.1002 / \mathrm{hrm} .20434$.

Damásio, B. F. (2012). Uso da análise fatorial exploratória em psicologia. Avaliação Psicológica, 11(2), 213-228. Recuperado de pepsic.bvsalud. org/pdf/avp/v11n2/v11n2a07.pdf

Franco, K. S., Neiva, E. R., Nery, V. F., \& Demo, G. (2016). The relationship between context, attitudes and well-being in organizational change. Psicologia: Teoria e Pesquisa, 32(n. esp.), 1-10, doi: 10.1590/0102-3772e32ne219

George, J. M., \& Jones, G. R. (2001). Towards a process model of individual change in organization. Human Relations, 54(4), 419-444. Recuperado de pepsic.bvsalud.org/pdf/avp/v11n2/v11n2a07.pdf

Herscovitch, L., \& Meyer, J. P. (2002). Commitment to organizational change: Extension of a three-component model. Journal of Applied Psychology, 87(3), 474-87. doi: 10.1037/0021-9010.87.3.474

Hox, J. J. (2010). Multilevel analysis. Techniques and applications (2 ed). New York: Routledge.

Huck, S. W. (2009). Statistical misconceptions. London: Psychology Press.

Kim, T. G., Hornung, S., \& Rousseau, D. M. (2011). Change-supportive employee behavior: antecedents and the moderating role of time. Journal of Management, 37, 1664-1693. doi: 10.1177/0149206310364243

Klein, K., \& Kozlowski, S. (2000). Multilevel theory, research and methods in organizations. San Francisco: Jossey-Bass.

Lines, R. (2005). The structure and function of attitudes toward organizational change. Human Resource Development Review, 4(1), 8-32. doi: $10.1177 / 1534484304273818$

Lysova, E. I., Richardson, J., Khapova, S. N., \& Jansen, P. G. W. (2015). Change supportive employee behavior: A career identity explanation. Career Development International, 20(1), 38-62. doi: 10.1108/CDI-03-2014-0042

Mardia, K. V. (1971). The effect of nonnormality on some multivariate tests and robustness to nonnormality in the linearmodel. Biometrika, 88(1), 105-121. doi: 10.1093/biom-et/58.1.105

Neiva, E. R., \& Paz, M. G. T. (2012). Percepção de mudança individual e organizacional: O papel das atitudes, dos valores, do poder e da capacidade organizacional. Revista de Administração (FEA-USP), 47, 22-37. Recuperado de http://www.scielo.br/pdf/rausp/v47n1/v47n1a02

Nery, V. F., \& Neiva, E. R. (2015). Variáveis de contexto e respostas à mudança organizacional: testando o papel mediador das atitudes. Psicologia: Teoria e Pesquisa, 31(2), 259-268. doi: 10.1590/0102-37722015021968259268

Oreg, S. (2003). Resistance to change: Developing an individual differences measure. Journal of Applied Psychology, 88(4), 680-693. doi: 10.1037/0021-9010.88.4.680

Oreg, S., Bayazit, M., Vakola, M., Arciniega, L., Armenakis, A., Barkauskiene, R., Bozoinelos, N., Fujimoto, Y., González, L., Han, J., Hrebícková, M., Jimmieson, N., Kordacová, J., Mitsuhashi, H., Mlacic, B., Feric, I., Topic, M., Kotrla, Ohly, S., Saksvik, P., Hetland, H., Saksvik, I. and van Dam, K. (2008). Dispositional resistance to change: Measurement equivalence and the link to personal values across 17 nations. Journal of Applied Psychology, 93(4): 935-944. doi: $10.1037 / 0021-9010.93 .4 .935$

Oreg, S., Vakola, M., \& Armenakis, A. (2011). Change recipients' reactions to organizational change: A 60-year review of quantitative studies. The Journal of Applied Behavioral Science, 47(4), 461-524. doi: 10.1177/0021886310396550

Pasquali, L. (2010). Testes referentes a construto: Teoria e modelo de construção. Em L. Pasquali (Ed.), Instrumentação Psicológica: Fundamentos e Prática (pp. 165-188). Porto Alegre: Artmed.

Peccei, R., Giangreco, A., \& Sebastiano A. (2011). The role of organizational commitment in the analysis of resistance to change: Copredictor and moderator effects. Personnel Review, 2, 185-204. doi: 10.1108/004834811111060

Piderit, S. K. (2000). Rethinking resistence and recognizing ambivalence: A multidimensional view of attitudes toward an organizational change. Academy of Management Review, 25(4), 783-794.

Rhemtulla, M., Brosseau-Liard, P.E., \& Savalei, V. (2012). "When Can Categorical Variables Be Treated as Continuous? A Comparison of Robust Continuous and Categorical SEM Estimation Methods Under Suboptimal Conditions." Psychological Methods, 17, 354-373. doi: 10.1037/a0029315

Tabachnick, B. G., \& Fidell, L. S. (2013). Using multivariate statistics. 6th Edition, Boston: Pearson International Education.

Vakola, M. (2016). The reasons behind change recipients' behavioral reactions: a longitudinal investigation. Journal of Managerial Psychology, 31(1), 202-215. doi: 10.1108/JMP-02-2013-0058

Vakola, M., Armenakis, A., \& Oreg, S. (2013). Reactions to organizational change from an individual differences perspective: A review of empirical research (95-122). Em S. Oreg, A. Mitchel \& R. Todnem By. (Editors). The psychology of organizational change: Viewing change from the employee's perspective. Cambridge: Cambridge University Press.

Wiltermuth, S. S., \& Heath, C. (2009). Synchrony and cooperation. Psychological Science, 20(1), 1-5.

recebido em janeiro de 2017 aprovado em outubro de 2017

\section{Sobre as autoras}

Vanessa de Fátima Nery é Doutora pelo Programa de Pós-graduação em Psicologia Social, do Trabalho e das Organizações da Universidade de Brasília.

Elaine Rabelo Neiva é professora do Programa de Pós-graduação em Psicologia Social, do Trabalho e das Organizações da Universidade de Brasília.

Kettyplyn Kamilla Sanches Franco é graduada em Administração e Comunicação Social, Mestre pelo Programa de Pós-graduação em Psicologia Social, do Trabalho e das Organizações da Universidade de Brasília. 